\title{
Introducing managerial attention allocation in incentive contracts
}

\author{
Ricard Gil · Jordi Mondria
}

Received: 3 February 2010 / Accepted: 4 April 2011 / Published online: 22 April 2011 (C) The Author(s) 2011. This article is published with open access at SpringerLink.com

\begin{abstract}
This paper introduces and studies the role of managerial attention allocation constraints in incentive contracts. We extend the traditional moral-hazard benchmark model with multi-tasking and linear incentive contracts by letting the principal choose the amount of monitoring allocated across tasks. In our model, more attention allocated to a task improves the task contractibility and consequently increases the effort provided by the agent. Our findings show that, even under symmetry, in the presence of increasing returns to scale in either production or monitoring the principal may optimally offer an unbalanced incentive contract while allocating different amounts of attention across tasks. Finally, we comment on the empirical content of our model.
\end{abstract}

Keywords Incentive contracts - Attention allocation - Rational inattention . Monitoring

JEL Classification D86 $\cdot$ L14

We would like to thank comments from Dongsoo Shin, Donald Wittman, Brian Silverman, Heikki Rantakari and Niko Matouschek as well as seminar participants at UC Santa Cruz, IIOC 2008 and ISNIE 2008. All remaining errors are ours.

R. Gil (凶)

UC Santa Cruz, Santa Cruz, CA, USA

e-mail: rgil@ucsc.edu

J. Mondria

University of Toronto, Toronto, Ontario, Canada 


\section{Introduction}

To this day, there is an extensive literature in economics that focuses on the study of contract theory and the principal-agent problem. Most of these papers start off by assuming the presence of a principal that hires an agent to undertake a certain action which is not perfectly observable. For this reason, the agent may not exert enough effort to take the action under fixed-wage contract due to moral hazard. On the other hand, under variable compensation she may be reluctant to exert the costly effort if she is concerned that she may not be rewarded due to the less-than-perfect action observability. The principal takes into account these factors and designs a contract that aligns the interests of the agent with those of their own. The optimal contract will provide stronger incentives the more observable the actions are and the lower the agent's degree of risk aversion. These predictions were first formalized by Holmstrom (1979) and Shavell (1979) for the case of one-task jobs and by Holmstrom and Milgrom (1991) in the case of jobs with many tasks. In these papers and most of the previous literature, the main role of the principal is to design an optimal incentive contract for the agent while taking the contractibility of actions as given. ${ }^{1}$ In this paper we extend the existing framework from Holmstrom and Milgrom (1991) and (1994) to allow for endogenous task contractibility as we introduce the role of attention allocation constraint in incentive contracts. The goal of this paper is then to derive the optimal incentive contracts in a setting where the principal shapes the contracting environment by simultaneously choosing monitoring intensity and designing the incentive contract that she will offer to the agent.

For this purpose, in this paper we build upon the multi-tasking contracting scenario in Holmstrom and Milgrom (1991) and (1994) by introducing an attention allocation constraint on the principal/manager side. The model presents a risk-neutral principal contracting with a risk-averse agent over effort in two different tasks. The agent's effort is not perfectly observable and thus the moral hazard problem. We introduce to the classic contracting problem an attention constraint for the manager. The principal is endowed with a monitoring capacity that must be allocated among all tasks that define the job. This monitoring capacity can adopt the form of investment in monitoring technology or actual time spent by the manager monitoring and watching employees. The problem of the manager then becomes how much of the attention capacity to allocate to each task. More attention allocated to a given task increases the precision (decreases the uncertainty) of pay for performance measures in the given task used by the principal in the incentive contract, and therefore mitigates the moral hazard problem. Since the principal is constrained on the total level of attention that she can allocate among all tasks, she faces a trade-off on effort measurability across tasks. In other words, in this paper the principal's monitoring decisions are endogenous and that affects the contractibility of the tasks that define the job. This differs from previous research in that typically the monitoring decisions of the principal are implicitly taken as given and exogenously changed to analyze comparative statics off the equilibrium.

\footnotetext{
1 Some papers (such as Lucas 1978) have allowed the principal to take costly actions that enhance the profitability of the agent's actions.
} 
We solve the model through backward induction where the only difference with the regular model is that in the first period the principal chooses the allocation of her attention endowment across all tasks. Our solution generates two main results that we describe next. We first examine the reaction of the agent to incentives and show consistent results with the previous literature. An increase in the attention allocated to one task leads to a decrease in the uncertainty of the pay for performance measure and an increase in the contractibility of that task. As a consequence, the agent will increase the effort exerted in that task due to the decrease in the uncertainty of the pay for performance measure. This comes as no surprise since the innovation in our paper comes from the principal side and we leave unchanged the agent side relative to other papers. This result also shows that a strong complementarity between attention allocation and incentive provision emerges endogenously within the model.

This strong complementarity between incentive provision and monitoring intensity is endogenous in our model and is at odds with conventional results from Hennart (1993) where managers may decide to monitor some tasks and provide incentive contracts for others. Our results show that in particular cases of a multi-task setting these tools are complementary (instead of substitutes).

Once established the link between managerial attention and the effort of the agent, our second set of results follows. Our model allows us to investigate how the manager allocates the monitoring capacity across tasks under different circumstances and how she optimally coordinates incentive provision across tasks within a contract and the allocation of her attention endowment. For this purpose, we compare results under different technologies in production and monitoring. We find that the principal will optimally allocate the same level of managerial attention and provide the same incentives across tasks when we impose symmetric decreasing returns to scale in production and monitoring. Not surprisingly, the principal or manager will also choose an asymmetric allocation of attention when there is an asymmetry in the monitoring technology or in the contribution to profits of each task. However, even in the case of symmetric technologies across tasks, there may be an optimal asymmetric attention allocation and incentive provision across tasks when there are increasing returns to scale to either production or monitoring.

To the best of our knowledge, the framework and results in this paper are a contribution to this literature for a variety of reasons. On the content side of the paper, we believe it provides a more realistic view of the manager's role in agency relationships by showing how managers interact and combine incentive contracts with monitoring. ${ }^{2}$ To our knowledge, two papers are closest to ours. Demougin and Fluet (2001) examine the trade-off between incentives and monitoring within a firm cost minimization problem. Consistent with our results, they find that incentives and monitoring may be complementary under certain circumstances. Their approach differs from ours in that their monitoring technology helps repairing the agent's mistakes within a poisson technology and ours focuses on the standard moral hazard problem introduced

\footnotetext{
2 Our definition of the monitoring technology may be too narrow. Mainly the problem presented here establishes the fact that the principal/manager is constraint on how much monitoring she can undertake. Therefore the total capacity of attention may take the form of time, money investment or monitoring technology or personel that can be distributed among all tasks of production.
} 
in Holmstrom and Milgrom (1991). The second paper close to ours is Khalil and Lawarree (1995) where they show that there is a complementarity between monitoring type (input versus output monitoring) and incentive provision. Other papers have also examined similar topics. A few examples are MacDonald and Marx (2001) who highlight the importance of complementarities between task composition and incentive contracts taking the contractibility of effort as exogenous; Kessler (2004) where managers are allowed to optimize between quantity and quality of monitoring; or Lazear (2006) who also shows that attention allocation by the principal may lead agents to particularly focus on certain aspects of the tasks they perform and in response to that the principal may choose to focus on specific tasks. On the other hand, this paper represents the first application of the ideas of the inattentiveness literature started off by Mankiw and Reis (2002) and Sims (2003) and (2006) to optimal incentive contracts.

Finally, on the empirical content of the model, our findings sheds light on possible biases on estimates offered by previous papers as up to now the empirical literature has not taken into account differences in monitoring technologies across firms and jobs. We discuss the implications of the absence of such controls and guide future research characterizing the ideal data set that will allow testing the predictions of our model (as opposed to those of others) easier. Our model displays a complementarity between incentives and attention and therefore the main empirical implication is that managers choose to provide higher incentives in those tasks that they simultaneously choose to monitor more closely. More specifically, it becomes key for researchers and econometricians to observe investments in monitoring along with incentive provision. When a shock occurs that causes the managers to redesign their incentive contracts we should observe a positive correlation between incentives and monitoring within tasks and a negative correlation between incentives and monitoring across tasks within a contract due to the attention allocation constraint. On the other hand, when managers are able to increase their attention constraint, we should observe an increase in monitoring and incentives across all tasks.

Unfortunately, prior literature in empirical contracting contains no evidence in monitoring intensities and therefore we find limited direct evidence at the moment that confirms the testable implications from our model. Hueth et al. (1999) is a rare exception that shows a positive correlation between incentive provision and monitoring in contracts between growers and first handlers in California fruit and vegetable markets. Other papers provide indirect evidence consistent with our implications. Baker and Hubbard (2003) document the impact of an exogenous change in monitoring costs due to the introduction of on-board computers in the trucking industry. Lower monitoring costs increased the amount of monitoring by trucking companies and the use of outsourcing (as opposed to in-house contracting) in shipping services.

The paper is structured as follows. Section 2 relates this paper to the relevant preceding literature. Section 3 presents and solves the model under symmetric task technologies. This section shows how the introduction of managerial attention allocation shapes the design of optimal incentive contracts. In Sect. 4, we consider the introduction of asymmetries between tasks and we obtain that results do not change qualitatively. We also extend our model in Sect. 5 by adding complementarities in production across tasks. Finally, Sect. 6 discusses the empirical content of our model 
and comments on existing supporting evidence in the empirical contracting literature and Sect. 7 concludes.

\section{Literature review}

This paper builds on and contributes directly to two different economic literatures. These are the literature on optimal incentive contracts and its recent stream of papers departing from the standard rationality assumptions, and the literature on attention allocation that has been mainly developed in macroeconomics and only now recently applied to other fields in economics.

\subsection{Literature on optimal incentive contracts}

The literature on optimal incentive contracts is extensive and confronts many and very different types of information asymmetries. Here we review the literature on incentive contracts dealing with moral hazard issues and risk aversion. Among the first contributors to this literature are Holmstrom (1979) and Shavell (1979) who studied the case of one-dimensional effort problems, and Holmstrom and Milgrom (1991) who extended the literature by examining multi-dimensional effort problems. The former papers established the optimality of the negative relationship between uncertainty and incentive intensity while the latter emphasized the necessity of balancing incentives across tasks and the importance of job design. Following this literature, others have studied the distortion of performance measures in incentive contracts (Baker 1992) or the role of subjective pay for performance (Baker et al. 1994) in the optimal design of incentive contracts. Yet most studies take the contractibility of effort as given assuming an uncertainty variance-covariance matrix $\Sigma$.

In this paper, we introduce a managerial attention allocation constraint and relax the agent's effort constraint by assuming a convex disutility of effort. This novelty allows us to endogeneize the up-to-now exogenous variance-covariance matrix and therefore explore how the manager balances the use of incentives and attention across tasks. Other papers before ours have modelled the endogenous decision of monitoring (Demougin and Fluet 2001; Khalil and Lawarree 1995; MacDonald and Marx 2001; Kessler 2004), but in our model monitoring decisions do not enter directly the principal's profit function and yet mitigate the moral hazard problem in a multi-tasking setting. A direct consequence of this novelty of our approach is that managers may decide to mute incentives in one task and concentrate their attention and incentive provision into another task. There are other papers that document cases where a principal optimally chooses leaving a task outside a formal contract and therefore choosing the degree of contractual completeness (see Holmstrom and Milgrom 1994; Corts 2007 regarding job design and Hart and Moore 2004 or Wernerfelt 2007 for endogenous contractual completeness among others). Our approach here differs from those in that our principal faces a trade-off between decreasing the contractibility of a task and increasing the contractibility of another task and therefore we are very precise about the nature of the cost incurred by the principal.

This paper also contributes to a recent stream of papers that has introduced into the analysis of optimal contracting new elements that depart from traditional rationality 
assumptions. See recent examples of this literature in Hart and Moore (2008) bringing entitleness into bargaining or Fehr and others ${ }^{3}$ examining the consequences of inequity aversion or reciprocity in optimal incentive contracts. Our paper differs from these in that managers in our framework are self-interested but instead of working around the contractibility shortcomings we allow for an endogenous solution to the degree of effort contractibility.

\subsection{Literature on attention allocation}

This paper is not the first to apply inattentiveness in economics, but it is, to the best of our knowledge, among the first papers to examine the role of inattentiveness in contracting while endogeneizing the degree of effort contractibility within a standard moral hazard model. Gifford (2004) derives a model of make-or-buy decisions and endogenous transaction costs with attention allocation. Her paper follows the transaction cost economics approach to explaining make-or-buy decisions and therefore assumes that contractual incompleteness of tasks performed inside the firm are unimportant since all distortions can be taken care of within the firm. Our approach here differs from hers in that the source of contract distortion (i.e., moral hazard) remains inside the firm and we examine the role of attention allocation in dealing with employment contracts within a firm.

This paper also relates to the recent literature on attention allocation and inattentiveness. Inattentive agents have been used in several fields. In macroeconomics, inattention has explained sticky prices in Mankiw and Reis (2002) and Mackoviak and Wiederholt (2007) and consumption dynamics in Reis (2006a,b) and Luo (2007). In finance, attention allocation decisions have been used to understand financial contagion across emerging economies in Mondria (2006) and portfolio under-diversification in Van Nieuwerburgh and Veldkamp (2007a). In international finance, inattentive investors help explain the forward discount puzzle in Bacchetta and Van Wincoop (2006) and the home bias puzzle in Van Nieuwerburgh and Veldkamp (2007b).

Despite the novelty of our approach, we recognize that previous research has characterized the main role of the principal as one of allocating resources across workers or tasks or even choosing the optimal number of workers being managed by one sole manager. Lucas (1978) shows that higher skill workers are more likely to become managers and are more likely to manage bigger firms. His result speaks about the distribution of firm sizes in the economy, but does not focus into the attention allocation constraint of the managers. Similarly, Rosen (1982) examines the allocation of talent within the hierarchy of a firm and across firms within the economy. He shows how more skilled managers should be solving more important problems and therefore located in higher up positions in the hierarchy of bigger firms. His result also focuses on the distribution of the size of firms and the distribution of earnings in the economy. For both these papers (and the literature that followed) higher skilled managers are allocated to more important problems to maximize revenues and therefore the same principle that drives the introduction of attention allocation is at use. Our approach

\footnotetext{
3 See Fehr and Schmidt (2003), Fehr et al. (2007), and Englmaier and Wambach (2007) among others.
} 
differs from all these in that in our paper attention allocation helps monitoring tasks and increases the contractibility of effort exerted on a given task by increasing the precision of the effort on that task.

\section{The model}

In this section we present our model as an extension to the framework in Holmstrom (1979) and Holmstrom and Milgrom (1991). This model presents a principal contracting over the non-contractible multidimensional effort of an agent. The agent provides effort in a number of tasks and the principal designs a linear contract, composed by a variable and a fixed factor, and monitors the effort of the agent. Since the effort in each task is not contractible, the principal writes an incentive contract contingent on some public (observable to a third party and contractible) signal non-perfectly correlated with effort. We present here the benchmark model with two tasks. ${ }^{4}$

\subsection{Benchmark description}

This model presents a principal contracting over the non-contractible effort provided by an agent in two tasks. The agent chooses a vector of efforts $t=\left(t_{1}, t_{2}\right)$, which are not directly observed by the principal. The agent faces a personal $\cos t C\left(t_{i}\right)=\frac{1}{2} t_{i}^{2}$ for a level of effort $t_{i}$ in each task $i$. Since the principal cannot observe the effort provided in each task, $t_{i}$, directly (effort in task is not contractible, she may be able to observe it but there is no third party that can), she writes an incentive contract contingent on some public (observable to a third party and contractible) signal $x_{i}$ correlated with the effort $t_{i}$ such that

$$
x_{i}=t_{i}+\epsilon_{i} \text { for each task } i
$$

where $\epsilon_{i}$ is normally distributed with mean 0 and variance $\sigma_{i}^{2}$, and $\epsilon_{1}$ is independent of $\epsilon_{2}$. The principal designs (assume) a linear contract composed by a variable and a fixed factor. The agent receives a total compensation of $w(X)=\beta+\alpha^{T} X$ where $\alpha=\left(\alpha_{1}, \alpha_{2}\right)^{\prime}$ is the vector of incentive intensity for each task and $X=\left(x_{1}, x_{2}\right)^{\prime}$ is a vector of observable signals about the effort provided by each agent. The agent, with an absolute coefficient of risk aversion $r$, has CARA preferences over the total compensation such that $u(w)=-e^{-r w}$. On the other hand, the principal is risk neutral. The efforts $t_{i}$ provided by the agent generate a private gross expected profit to the principal

$$
B\left(t_{1}, t_{2}\right)=t_{1}^{\theta}+t_{2}^{\theta}
$$

This gross expected profit function is flexible enough to provide decreasing, constant and increasing returns to each task depending on the value of the parameter $\theta$.

\footnotetext{
4 The case presented here is easily generalizable to the case of $n$ tasks.
} 
In this model, unlike the rest of the literature, the principal is able to decide how much monitoring she wants to do about the effort provided in each task. ${ }^{5}$ The principal would like to observe a signal that reduces all the uncertainty about the effort. However, the principal faces a technological constraint on monitoring, which is called attention allocation constraint. The principal is assumed to be endowed with $\kappa$ units of monitoring capacity, which needs to allocate to both tasks such that

$$
\kappa=\kappa_{1}+\kappa_{2}
$$

where the monitoring technology for each task $i$ is given by $\sigma_{i}^{2}=\frac{1}{\kappa_{i}^{\phi}}$. The more attention is allocated to one task, the less uncertainty about the effort provided by the agent about that particular task. The monitoring technology is flexible enough to provide decreasing, constant and increasing returns to the attention allocated to a particular task depending on the value of the parameter $\phi$. This constraint restricts the amount of information that the principal can process about the efforts that the agent is providing. This restriction could be interpreted as the principal having a limited amount of time to concentrate on monitoring the agent. The principal faces a trade-off between how much monitoring to allocate to each task. The principal cannot allocate negative attention to any task, which means $\kappa_{i} \geq 0$ for any $i$. The benchmark case assumes away complementarities between tasks (in both production and monitoring technologies).

\subsection{Model solution}

The model is solved using backward induction. First, for a given wage contract $(\alpha, \beta)$ and managerial attention allocation, $\left(\kappa_{1}, \kappa_{2}\right)$, the agent chooses the effort, $t$, she wants to provide in each task. Second, given the optimal effort of the agent, the principal chooses the wage contract for any managerial attention allocation. Third, given the optimal effort and the optimal wage contract, the principal chooses the optimal managerial attention allocation.

Following Holmstrom and Milgrom (1991), since the contract wage is normally distributed, the agent's certainty equivalent can be written by

$$
C E=\alpha^{T} t+\beta-C(t)-\frac{1}{2} r \alpha^{T} \Sigma \alpha
$$

where $\Sigma$ is the diagonal matrix of the vector of error terms in the private signal $\left(\epsilon_{1}, \epsilon_{2}\right)^{\prime}$. For a given wage contract $(\alpha, \beta)$ and managerial monitoring technology $\left(\kappa_{1}, \kappa_{2}\right)$, which implies a given $\Sigma$, the agent optimally chooses an effort in each task that is given as

$$
t_{i}=\alpha_{i}
$$

\footnotetext{
5 Amount of monitoring in this model can be actual direct monitoring attention or money spent on technology to monitor a certain task.
} 
The principal expected profits are given by $B(t)-\alpha^{T} \mu(t)-\beta$. Since the principal is risk neutral, she chooses the wage contract and the managerial attention allocation to maximize the following joint certainty equivalent of the principal and the agent (their joint surplus) for an optimal effort provided by the agent

$$
\max _{\left\{\alpha_{i}, \kappa_{i}\right\}_{i=1}^{2}} B(t)-C(t)-\frac{1}{2} r \alpha^{T} \Sigma \alpha \text { subject to } t_{i}=\alpha_{i}, \quad \kappa=\kappa_{1}+\kappa_{2}, \quad \kappa_{1} \geq 0, \quad \kappa_{2} \geq 0
$$

As Holmstrom and Milgrom (1991) noted, the joint surplus is independent of the intercept $\beta$ that is used to distribute the joint certainty equivalent between both parties. Thus, the optimal incentive intensity provided by the principal is given by

$$
\alpha_{i}=\left[\frac{1}{\theta}\left(1+\frac{r}{\kappa_{i}^{\phi}}\right)\right]^{\frac{1}{\theta-2}}
$$

as long as $\theta<2$. If $\theta \geq 2$, there would be a corner solution with zero or infinite effort. This is due to the assumption of quadratic costs to effort by the agent and the attention allocation constraint of the manager. The more attention allocated to one task, the higher is the incentive intensity the principal offers and the higher the effort the agent provides on that task. This result shows that the principal has a complementarity between the attention allocated to a task and the incentive intensity of that task. Since incentive design and attention allocation are the two tools through which the principal maximizes profits, this complementarity conditions the decision in each task.

The objective function for the managerial attention allocation optimization problem given the optimal effort and the optimal wage contract is obtained by plugging the optimal incentive intensity, $\alpha$, in Eq. (3) provided by the principal and the optimal effort, $t$, provided by the agent in Eq. (2) into the joint certainty equivalent. Once this is done, the principal's managerial attention allocation is obtained by maximizing the principal's objective function in terms of $\kappa_{i}$ such that

$$
\max _{\left\{\kappa_{i}\right\}_{i=1}^{2}} \sum_{i=1}^{2} A\left(1+\frac{r}{\kappa_{i}^{\phi}}\right)^{\frac{\theta}{\theta-2}} \text { subject to } \kappa=\kappa_{1}+\kappa_{2}, \kappa_{1} \geq 0, \kappa_{2} \geq 0
$$

where $A=\left[\left(\frac{1}{\theta}\right)^{\frac{\theta}{\theta-2}}-\frac{1}{2}\left(\frac{1}{\theta}\right)^{\frac{2}{\theta-2}}\right]$, which is always a strictly positive function as long as $\theta<2$.

Proposition 1 The symmetric managerial attention allocation $\kappa_{1}=\kappa_{2}=\frac{\kappa}{2}$ is a strict local maximum if and only if the following parameter constraint is satisfied

$$
\left(1+\frac{1}{\phi}\right)\left(\frac{\left(\frac{\kappa}{2}\right)^{\phi}}{r}+1\right)>\frac{2}{2-\theta}
$$

Proof If we introduce the attention allocation constraint from Eq. (1) into the objective function in Eq. (4), we obtain the following maximization problem 


$$
\max _{\kappa_{1}} A\left[\left(1+\frac{r}{\kappa_{1}^{\phi}}\right)^{\frac{\theta}{\theta-2}}+\left(1+\frac{r}{\left(\kappa-\kappa_{1}\right)^{\phi}}\right)^{\frac{\theta}{\theta-2}}\right]
$$

The second order condition of this problem when there is a symmetric attention allocation such that $\kappa_{1}=\kappa_{2}=\frac{\kappa}{2}$ is given by

$$
\frac{\partial^{2}}{\partial \kappa_{1}^{2}}\left(\frac{\kappa}{2}\right)=(\text { strictly negative constant })\left[-\left(\frac{2}{2-\theta}\right)+\left(1+\frac{1}{\phi}\right)\left(\frac{\left(\frac{\kappa}{2}\right)^{\phi}}{r}+1\right)\right]
$$

which is negative if and only if

$$
-\left(\frac{2}{2-\theta}\right)+\left(1+\frac{1}{\phi}\right)\left(\frac{\left(\frac{\kappa}{2}\right)^{\phi}}{r}+1\right)>0
$$

Note that through all the paper we are also assuming that $\theta<2$.

The inequality condition in Proposition 1 implies that a symmetric managerial attention allocation is optimal when the total monitoring capacity $\kappa$ is large, the agent's degree of risk aversion $r$ is low, the returns to scale in effort $\theta$ (in the gross expected profit function) and the returns to scale in monitoring $\phi$ are low (provided the monitoring capacity $\kappa$ is not too large). We explain the intuition behind each one of these results next.

If the principal is endowed with a large monitoring capacity $\kappa$, the attention allocation decision loses relevance since the principal is less attention constrained and therefore the problem at hand is less of a concern. A second implication from Proposition 1 above is that a lower degree of risk aversion (low $r$ ) implies that the agent is less sensitive to uncertainty and therefore the principal will have more incentives to allocate the same attention to both tasks. Our results also predict that the lower the returns to scale in the expected profits (lower $\theta$ ) the larger is the set of parameter values for which the principal allocates the same amount of monitoring to both tasks. This is so because the principal's marginal benefit to accumulating attention in one task decreases sharply. For this reason, the principal equally motivates both tasks and therefore allocates the same attention to both tasks. Finally, if there are low returns to scale in the monitoring technology, low $\phi$, the principal allocates attention to the effort provided by the agent in both tasks for exactly the same reason as when we have low returns to scale in production (low $\theta$ ).

Corollary 1 The symmetric managerial monitoring attention allocation $\kappa_{1}=\kappa_{2}=$ $\frac{\kappa}{2}$ is a unique global maximum if the following monitoring parameter constraint is satisfied

$$
\left(1+\frac{1}{\phi}\right)>\frac{2}{2-\theta}
$$


Proof The first order condition to the maximization problem in Eq. (5) equals zero if

$$
\kappa_{1}^{1+\phi}\left(1+\frac{r}{\kappa_{1}^{\phi}}\right)^{\frac{2}{2-\theta}}=\left(\kappa-\kappa_{1}\right)^{1+\phi}\left(1+\frac{r}{\left(\kappa-\kappa_{1}\right)^{\phi}}\right)^{\frac{2}{2-\theta}}
$$

The left hand side (LHS) is a continuous and strictly increasing function of $\kappa_{1}$ for $\kappa_{1} \geq 0$ and the right hand side (RHS) is a continuous and strictly decreasing function of $\kappa_{1}$ for $\kappa_{1} \geq 0$ if the following parameter constraint is satisfied

$$
\left(1+\frac{1}{\phi}\right)>\frac{2}{2-\theta}
$$

Hence, the first order condition equals zero given in Eq. (7) has a unique solution $\kappa_{1}=\frac{\kappa}{2}$. The first order conditions are strictly positive for $\kappa_{1} \in\left[0, \frac{\kappa}{2}\right)$ and strictly negative for $\kappa_{1} \in\left(\frac{\kappa}{2}, \kappa\right]$. The second order condition in Eq. (6) at $\kappa_{1}=\frac{\kappa}{2}$ is always negative. Therefore, the symmetric managerial monitoring attention allocation $\kappa_{1}=\kappa_{2}=\frac{\kappa}{2}$ is a unique global maximum when the sufficient parameter constraint is satisfied.

The result of Corollary 1 states that a simple comparison between the returns to scale in production and monitoring is enough to determine if allocating the same amount of attention to both tasks, $\kappa_{1}=\kappa_{2}=\frac{\kappa}{2}$, is a global maximum. This condition establishes that the lower the returns to scale to production, $\theta$, and to monitoring, $\phi$, the larger is the set of parameter values for which the principal allocates the same amount of resources to the monitoring of both tasks. The reason follows from our comments above on the condition in Proposition 1. If the returns to scale in production and monitoring are low enough, the marginal benefit to accumulating attention in any of the tasks and consequently focus production in that task will also be low to the point that the principal will be better off reallocating some of the attention across tasks and benefit from an increase in the marginal benefit to attention in both tasks.

Corollary 2 There exist asymmetric managerial monitoring attention allocation equilibria if the following parameter constraint is satisfied

$$
\left(1+\frac{1}{\phi}\right)\left(\frac{\left(\frac{\kappa}{2}\right)^{\phi}}{r}+1\right)<\frac{2}{2-\theta}
$$

Proof If the constraint is satisfied, the symmetric managerial attention allocation $\kappa_{1}=$ $\kappa_{2}=\frac{\kappa}{2}$ is a strict local minimum. The objective function is a continuous function over a compact set $\kappa_{1} \in[0, \kappa]$, hence there exist a maximum and a minimum. Furthermore, since the objective function in Eq. (5) is a symmetric function around $\kappa_{1}=\frac{\kappa}{2}$, there exits at least two asymmetric equilibria where one type of equilibria is such that $\kappa_{1}>\frac{\kappa}{2}>\kappa_{2}$ and the other type of equilibria is such that $\kappa_{1}<\frac{\kappa}{2}<\kappa_{2}$.

Finally, the second and last corollary shows that even in our very simple setting where the tasks enter symmetrically in the principal and agent's problem, asymmetric attention allocation and incentive provision across tasks can result in equilibrium. 
Mainly, under sufficiently strong increasing returns to scale in production, $\theta$ close to 2 , or monitoring (as long as the total amount of monitoring capacity $\kappa$ is not too large), $\phi>1$, the principal optimally concentrates all her monitoring capacity in one task. This, in turn, strengthens the incentives for that task by increasing $\alpha$ and increasing the precision in which $t$ is measured. Under our functional assumptions on the monitoring technology, when the principal decides to allocate no attention to one of the tasks, the precision of that task decreases radically and therefore the principal is forced to set $\alpha=0$ for the task that is not being monitored. This result would change if we were to allow for a finite lower bound (similar to the Holmstrom and Milgrom (1991) framework where $\Sigma$ matrix is taken as given). In that case, the optimal incentive contract would entail $\alpha>0$ for all tasks where the value of some $\alpha^{\prime}$ s would be higher than others.

This result shows that in scenarios where jobs are defined by a number of differentiated tasks principals may still find optimal to provide unbalanced incentive contracts even if all tasks that define the job enter the principal's and the agent's objective functions symmetrically. Traditional explanations emphasized differences in the contractibility or the returns to scale across tasks as the main reason for observing asymmetries of incentive provision in these contracts. Here we show that in our model even under total symmetry among tasks, a principal may find optimal to mute incentives in a task to strengthen incentives in the other task. ${ }^{6}$ This result is driven by the main contribution in this paper, which is to allow the manager to optimally choose how to allocate her monitoring activities across tasks. ${ }^{7}$ These monitoring activities have a positive impact in the productivity of individuals since they increase the precision at which effort on a given task is measured. Symmetric increasing returns to scale in production or monitoring increase the set of parameters under which the principal provides an optimal asymmetric attention allocation across tasks and the consequent unbalanced provision of incentives.

For expositional purposes we illustrate the inequality of Proposition 1 (and Corollary 2) in Fig. 1 for an agent with a coefficient of risk aversion, $r=2$, and a principal with three different monitoring capacities, $\kappa=\{0.2,0.5,1\}$. The line in the graph divides the region of parameters where the principal monitors both tasks from the region of parameters where all the attention is allocated to one of the tasks. In the region of parameters $(\phi, \theta)$ above the line, the principal specializes in the monitoring of one of the tasks. Specialization arises if there are increasing returns to scale either in production or monitoring. It is important to highlight that when there are decreasing returns to scale in both production and monitoring, the principal always decides to allocate the same attention to both tasks. Similarly, the figure shows that neither increasing returns in production nor monitoring are sufficient conditions for specialization, but only necessary conditions. Finally, see that increasing returns in

\footnotetext{
6 This result would generalize easily to the case of more than two tasks.

7 It is important to acknowledge that our model relies on functional form assumptions that yield closed form solutions. Despite this, these functional forms have been used before in the literature. The only novelty in this paper may be our monitoring technology (which affects output variance around the agent's effort) and even the assumption on that functional form is rather innocuous.
} 
Fig. 1 This figure plots the combination of parameters $(\phi, \theta)$ such that the principal is indifferent between allocating monitoring capacity to both tasks or specializing when the monitoring capacity is given by $\kappa=0.2,0.5,1$ and the coefficient of risk aversion is given by $r=2$. Any combination of parameters $(\phi, \theta)$ above the line implies that the principal specializes in monitoring one task, while in any combination of parameters $(\phi, \theta)$ below the line, the principal allocates the same attention to both tasks

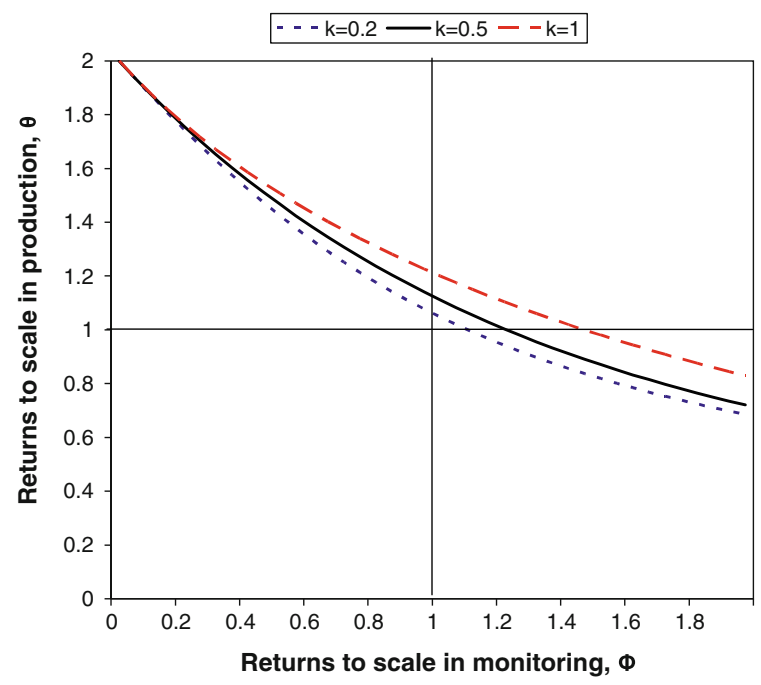

both technologies may not be enough since the three lines depicted in Fig. 1 are bounded away from the Cartesian point $(1,1)$.

\section{Introducing asymmetries between tasks}

The benchmark case above assumes symmetry across tasks in the gross expected profit function and the monitoring technology. In this section, we relax this symmetry assumption and introduce asymmetries first in the gross expected profit function and then in the monitoring technology. We aim to understand how sensitive our previous results are to the symmetry assumption and at the same time examine how our results compare to traditional results in the incentive contract literature when asymmetries across tasks exist.

\subsection{Asymmetry in the principal's expected profits}

The benchmark model assumes that both tasks provided by the agent generate the same gross expected profit to the principal. In this section, we show the optimal monitoring attention allocation when the tasks generate asymmetric gross expected profits to the principal. Assume that for the same amount of effort in both tasks, the principal receives a higher expected profit from the second task such that the gross expected profits of the principal are given by

$$
B\left(t_{1}, t_{2}\right)=t_{1}^{\theta}+\tau t_{2}^{\theta}, \text { where } \tau>1
$$

The optimal effort decision by the agent is not distorted and is still given by Eq. (2) from the benchmark model. However, the incentive intensity chosen by the principal is affected since her gross expected profits have changed. The principal chooses the 
wage contract and the managerial monitoring technology to maximize the following joint certainty equivalent of the principal and the agent (their joint surplus) for an optimal effort provided by the agent

$$
\max _{\left\{\alpha_{i}, \kappa_{i}\right\}_{i=1}^{2}} B(t)-C(t)-\frac{1}{2} r \alpha^{T} \Sigma \alpha \text { subject to } t_{i}=\alpha_{i}, \quad \kappa=\kappa_{1}+\kappa_{2}, \quad \kappa_{1} \geq 0, \quad \kappa_{2} \geq 0
$$

The optimal incentive intensity provided by the principal is given by

$$
\alpha_{1}=\left[\frac{1}{\theta}\left(1+\frac{r}{\kappa_{1}^{\phi}}\right)\right]^{\frac{1}{\theta-2}}, \quad \alpha_{2}=\left[\frac{1}{\tau \theta}\left(1+\frac{r}{\kappa_{2}^{\phi}}\right)\right]^{\frac{1}{\theta-2}}
$$

as long as $\theta<2$. The optimal managerial monitoring attention allocation given the optimal effort and the optimal wage contract by the principal is obtained by plugging the optimal incentive intensity, $\left(\alpha_{1}, \alpha_{2}\right)$, provided by the principal and the optimal effort, $t$, provided by the agent into the joint certainty equivalent

$$
\begin{gathered}
\max _{\left\{\kappa_{i}\right\}_{i=1}^{2}} A\left(1+\frac{r}{\kappa_{1}^{\phi}}\right)^{\frac{\theta}{\theta-2}}+\tau^{\frac{2}{2-\theta}} A\left(1+\frac{r}{\kappa_{2}^{\phi}}\right)^{\frac{\theta}{\theta-2}} \\
\text { subject to } \kappa=\kappa_{1}+\kappa_{2}, \quad \kappa_{1} \geq 0, \quad \kappa_{2} \geq 0
\end{gathered}
$$

where $A=\left[\left(\frac{1}{\theta}\right)^{\frac{\theta}{\theta-2}}-\frac{1}{2}\left(\frac{1}{\theta}\right)^{\frac{2}{\theta-2}}\right]$, which is always a strictly positive function as long as $\theta<2$.

Corollary 3 There is a unique global maximum managerial monitoring attention allocation $\left(\kappa_{1}^{*}, \kappa_{2}^{*}\right)$ with $\kappa_{1}^{*}<\frac{\kappa}{2}<\kappa_{2}^{*}<\kappa$ when the tasks provide asymmetric gross expected profits (due $\tau>1)$ if the following parameter constraint is satisfied

$$
\left(1+\frac{1}{\phi}\right)>\frac{2}{2-\theta}
$$

Proof When we introduce the monitoring attention allocation constraint from Eq. (1) into the objective function in Eq. (8), the first order condition equals zero when

$$
\tau^{\frac{2}{2-\theta}} \kappa_{1}^{1+\phi}\left(1+\frac{r}{\kappa_{1}^{\phi}}\right)^{\frac{2}{2-\theta}}=\left(\kappa-\kappa_{1}\right)^{1+\phi}\left(1+\frac{r}{\left(\kappa-\kappa_{1}\right)^{\phi}}\right)^{\frac{2}{2-\theta}}
$$

The left hand side (LHS) is a strictly increasing function of $\kappa_{1}$ for $\kappa_{1} \geq 0$ and the right hand side (RHS) is a strictly decreasing function of $\kappa_{1}$ for $\kappa_{1} \geq 0$ if the following parameter constraint is satisfied $\left(1+\frac{1}{\phi}\right)>\frac{2}{2-\theta}$. If $\kappa_{1}=\frac{\kappa}{2}$, the LHS $>$ RHS. If $\kappa_{1}=0$, LHS $<$ RHS. Therefore, there exists a unique solution $\left(\kappa_{1}^{*}, \kappa_{2}^{*}\right)$ that makes RHS=LHS and $\kappa_{1}^{*}<\frac{\kappa}{2}<\kappa_{2}^{*}<\kappa$. The first order conditions are strictly positive for $\kappa_{1} \in\left[0, \kappa_{1}^{*}\right)$ and strictly negative for $\kappa_{1} \in\left(\kappa_{1}^{*}, \kappa\right]$. The second order condition 
at $\kappa_{1}^{*}$ is always negative. Therefore, the asymmetric managerial monitoring attention allocation $\left(\kappa_{1}^{*}, \kappa_{2}^{*}\right)$ is a unique global maximum when the parameter constraints $\left(1+\frac{1}{\phi}\right)>\frac{2}{2-\theta}$ and $\tau>1$ are satisfied.

In this case, the inequality condition in Corollary 3 shows that, due to the asymmetry in the gross expected profit function, the principal optimally allocates a bigger share of her monitoring capacity to the task with higher returns as long as both tasks exhibit the same degree of returns to scale. The incentive strength $\alpha$ placed to different tasks differs and the principal places $\alpha_{2}>\alpha_{1}$ provided that $\tau>1$. This result is consistent with the literature in that incentive contracts in multi-tasking settings optimally place stronger incentives on tasks that are more profitable to the principal.

Similarly to the results in the previous section, it is easy to see that in situations where the gross expected profit function or the monitoring technology exhibit increasing returns to scale (high $\theta$ or $\phi$ respectively), the principal chooses to allocate all the monitoring capacity in task $2\left(\kappa_{2}=\kappa\right)$ and place none in task $1\left(\kappa_{1}=0\right)$. Therefore, this extreme uneven allocation of attention would mute incentives in task $1\left(\alpha_{1}=0\right)$ and maximize incentives in task $2\left(\alpha_{2}>0\right)$. Again, we find that incentive contracts in a multi-tasking setting that mute incentives for one task may not come from differences in returns to effort but be a consequence of the optimal combination of attention allocation across tasks and the optimal provision of incentives. The principal understands that there is a complementarity between the provision of incentives and the allocation of attention in each task. In the presence of increasing returns to scale to effort in the gross expected profit function or in the monitoring technology, the marginal benefit of accumulating attention on a given task is greater than the marginal benefit of allocating attention to another task. In this case, the principal chooses to allocate all her monitoring capacity to a given task and write an optimal incentive contract that provides incentives to effort on one task only.

\subsection{Asymmetry in monitoring technology}

Similarly to the case presented above, the benchmark model assumes that monitoring both tasks cost the same. In this section, we show the optimal monitoring attention allocation when the tasks have different monitoring costs (due to different technologies). To proceed with this analysis, we assume that the second task requires more time of monitoring to reduce the same amount of uncertainty about the non-observable effort of the agent such that the attention allocation constraint is given by

$$
\kappa=\kappa_{1}+\tau \kappa_{2} \text {, where } \tau>1
$$

This constraint does not affect the optimal effort chosen by the agent in the benchmark model, which is given in Eq. (2), and it does not affect the optimal incentive intensity provided by the principal and given by Eq. (3) either. However the optimal monitoring attention allocation is distorted and this leads to our next proposition.

Corollary 4 There is a unique global maximum managerial monitoring attention allocation $\left(\kappa_{1}^{*}, \kappa_{2}^{*}\right)$ with $\kappa>\kappa_{1}^{*}>\frac{\kappa}{2}>\kappa_{2}^{*}$ when the tasks have different costs of monitoring $(\tau>1)$ if the following parameter constraint is satisfied 


$$
\left(1+\frac{1}{\phi}\right)>\frac{2}{2-\theta}
$$

Proof When we introduce the monitoring attention allocation constraint from Eq. (9) into the objective function in Eq. (4), the first order condition equals zero when

$$
\kappa_{1}^{1+\phi}\left(1+\frac{r}{\kappa_{1}^{\phi}}\right)^{\frac{2}{\theta-2}}=\tau \kappa_{2}^{1+\phi}\left(1+\frac{r}{\kappa_{2}^{\phi}}\right)^{\frac{2}{\theta-2}}
$$

where $\kappa_{2}=\frac{\left(\kappa-\kappa_{1}\right)}{\tau}$. The left hand side (LHS) is a strictly increasing function of $\kappa_{1}$ for $\kappa_{1} \geq 0$ and the right hand side (RHS) is a strictly decreasing function of $\kappa_{1}$ for $\kappa_{1} \geq 0$ if the following parameter constraint is satisfied $\left(1+\frac{1}{\phi}\right)>\frac{2}{2-\theta}$. If $\kappa_{1}=\kappa_{2}$, the LHS $<$ RHS. If $\kappa_{1}=\kappa$, LHS $>$ RHS. Therefore, there exists a unique solution $\left(\kappa_{1}^{*}, \kappa_{2}^{*}\right)$ that makes RHS $=$ LHS and $\kappa>\kappa_{1}^{*}>\frac{\kappa}{2}>\kappa_{2}^{*}$. The first order conditions are strictly positive for $\kappa_{1} \in\left[0, \kappa_{1}^{*}\right)$ and strictly negative for $\kappa_{1} \in\left(\kappa_{1}^{*}, \kappa\right]$. The second order condition at $\kappa_{1}^{*}$ is always negative. Therefore, the asymmetric managerial monitoring attention allocation $\left(\kappa_{1}^{*}, \kappa_{2}^{*}\right)$ is a unique global maximum when the parameter constraints $\left(1+\frac{1}{\phi}\right)>\frac{2}{2-\theta}$ and $\tau>1$ are satisfied.

When we consider the case that task 2 requires more units of monitoring capacity to increase precision of effort measurement by the same amount $(\tau>1)$, we find that the principal optimally allocates more attention to task 1 than to task $2\left(\kappa_{1}>\kappa_{2}\right)$. This asymmetric allocation of attention comes from the fact that to achieve equal precision across tasks the principal must allocate more units of attention to task 2 than to task 1. This means that at the margin the opportunity cost of the last unit of attention allocated to task 2 in terms of gains in precision of task 1 is higher than the increase in precision obtained in the measurement of effort exerted in task 2 . This unequal trade-off induces the principal to allocate more units of attention to task 1 up to the point at which the marginal gain in precision are equal across tasks and $\kappa_{1}>\kappa_{2}$. At this point, and given the existing complementarity between attention allocated and incentive provision to a task, the principal optimally chooses to provide stronger incentives to task 1 than to task $2\left(\alpha_{1}>\alpha_{2}\right)$.

This finding is indeed consistent with the main finding in Holmstrom (1979). Optimal incentive contracts mediating a risk-neutral principal and a risk-averse agent should provide stronger incentives for those tasks that are less costly to monitor. The novelty here is that the degree of monitorability is endogenous to the principal and she is able to combine that with the optimal incentive provision scheme.

Finally, and similarly to the previous section, under sufficiently strong increasing returns to scale in the production function or in the monitoring technology (high $\theta$ or $\phi$ respectively), the principal chooses to allocate all her attention to the task where attention is less costly (task 1 such that $\kappa_{1}=\kappa$ ) and allocate no attention to task $2\left(\kappa_{2}=0\right)$. In this case, the principal would offer a contract that offers no incentives for task $2\left(\alpha_{2}=0\right)$ and only provides incentives to task $1\left(\alpha_{1}>0\right)$. We want to point out that this corner result hinges on the specific functional assumption on the monitoring 
technology. In other words, there would be a positive provision of incentives to task $2, \alpha_{2}>0$, if we were to allow for a lower bound of precision.

The introduction of asymmetries in either the principal's expected profits or monitoring technology suggests that small variations in relative prices between tasks might lead to sharp changes in specialization patterns of firms. We will extend the discussion about this prediction in Sect. 6, where we discuss the empirical content of the model.

\section{Complementarity between tasks}

In the general framework above and the particular cases in the following sections, we assumed away any complementarity between tasks in production and monitoring. Next, we examine the case where the complementarity between tasks exists in the expected gross profit function, but still assuming away complementarities in the monitoring technology.

\subsection{Complementarity in the production function}

The benchmark model assumes that both tasks provided by the agent are independent of each other in generating gross expected profit to the principal. In this section, we show the optimal monitoring attention allocation when the tasks have strong complementarities. Assume that the expected gross profits by the principal are given by

$$
B\left(t_{1}, t_{2}\right)=t_{1}^{\theta} t_{2}^{\theta}
$$

This expected profit function introduces a strong complementarity between tasks because if one of the tasks is not provided by the agent, then the principal receives zero profits. The optimal effort decision by the agent is not distorted and is still given by Eq. (2) from the benchmark model. However, the incentive intensity chosen by the principal is affected since her gross expected profits have changed. The principal chooses the wage contract and the managerial monitoring technology to maximize the following joint certainty equivalent of the principal and the agent (their joint surplus) for an optimal effort provided by the agent

$$
\max _{\left\{\alpha_{i}, \kappa_{i}\right\}_{i=1}^{2}} t_{1}^{\theta} t_{2}^{\theta}-\frac{1}{2} \sum_{i=1}^{2} t_{i}^{2}\left(1+\frac{r}{\kappa_{i}^{\phi}}\right) \text { subject to } t_{i}=\alpha_{i}, \quad \kappa=\kappa_{1}+\kappa_{2}, \quad \kappa_{1} \geq 0, \quad \kappa_{2} \geq 0
$$

The optimal incentive intensity provided by the principal is given by

$$
\begin{gathered}
\alpha_{1}=\left[\frac{1}{\theta B^{\theta}}\left(1+\frac{r}{\kappa_{1}^{\phi}}\right)\right]^{\frac{1}{2 \theta-2}} \alpha_{2}=\left[\frac{1}{\theta B^{-\theta}}\left(1+\frac{r}{\kappa_{2}^{\phi}}\right)\right]^{\frac{1}{\theta-2}} \\
\text { where } B=\left(1+\frac{r}{\kappa_{1}^{\phi}}\right)^{\frac{1}{2}}\left(1+\frac{r}{\kappa_{2}^{\phi}}\right)^{-\frac{1}{2}}
\end{gathered}
$$


as long as $\theta<1$. If $\theta>1$, the principal could give the agent enough incentives to choose an infinite effort in both tasks. The optimal managerial monitoring attention allocation given the optimal effort and the optimal wage contract by the principal is obtained by plugging the optimal incentive intensity, $\left(\alpha_{1}, \alpha_{2}\right)$, provided by the principal and the optimal effort, $t$, provided by the agent into the joint certainty equivalent

$$
\max _{\left\{\kappa_{i}\right\}_{i=1}^{2}} A^{c}\left(1+\frac{r}{\kappa_{1}^{\phi}}\right)^{\frac{\theta}{2 \theta-2}}\left(1+\frac{r}{\kappa_{2}^{\phi}}\right)^{\frac{\theta}{2 \theta-2}} \text { subject to } \kappa=\kappa_{1}+\kappa_{2}, \quad \kappa_{1} \geq 0, \quad \kappa_{2} \geq 0
$$

where $A^{c}=\left[\theta^{\frac{\theta}{1-\theta}}-\theta^{\frac{1}{1-\theta}}\right]$, which is always a strictly positive function as long as $\theta<1$.

Proposition 2 There is a unique global maximum managerial monitoring attention allocation with $\kappa_{1}=\kappa_{2}=\frac{\kappa}{2}$.

Proof When we introduce the monitoring attention allocation constraint into the objective function in Eq. (10), the first order condition equals zero when

$$
\kappa_{1}^{1+\phi}\left(1+\frac{r}{\kappa_{1}^{\phi}}\right)=\left(\kappa-\kappa_{1}\right)^{1+\phi}\left(1+\frac{r}{\left(\kappa-\kappa_{1}\right)^{\phi}}\right)
$$

The right hand side (RHS) is a continuous and strictly increasing function of $\kappa_{1}$ for $\kappa_{1} \geq 0$ and the left hand side (LHS) is a continuous and strictly decreasing function of $\kappa_{1}$ for $\kappa_{1} \geq 0$. Hence, the first order condition equals zero has a unique solution $\kappa_{1}=\frac{\kappa}{2}$. The first order conditions are strictly positive for $\kappa_{1} \in\left[0, \frac{\kappa}{2}\right)$ and strictly negative for $\kappa_{1} \in\left(\frac{\kappa}{2}, \kappa\right]$. The second order condition is always negative. Therefore, the symmetric managerial monitoring attention allocation $\kappa_{1}=\kappa_{2}=\frac{\kappa}{2}$ is a unique global maximum.

Proposition 2 establishes the result that in the presence of sufficiently strong complementarities it will always be optimal to spread attention allocation across tasks since the introduction of a strong complementarity in the gross expected profit function of the principal eliminates the potential benefits of concentrating the principal's attention in any given task. In the particular scenario that we examine here, the principal optimally allocates equal attention to each task if each task exhibits decreasing returns to scale. This particular case has an odd but otherwise intuitive result given the assumptions of the model. If the task exhibit increasing returns to scale $(\theta>1)$ and the agent's internal cost of effort is quadratic, there will be no finite solution due to the complementarity among tasks. The principal benefits from increasing the agent's effort more than linearly even after compensating the agent above the cost of her effort.

\section{Empirical content of the model and supporting evidence}

In this section we review one by one the predictions from our model and comment on the ideal data set necessary to test these predictions and therefore the empirical 
validity of our model. Since the observation of monitoring practices is a key component of our model and traditional data sets lack information on this dimension, we comment on the resulting biases that emerge when current empirical analysis does not take into account variation in monitoring intensities across jobs and tasks. We complement this analysis by commenting on evidence in the literature consistent with our predictions.

As we advance in the previous paragraph, the findings in our model have several empirical implications that require of information on expenditures in monitoring technologies to be tested. The ideal data set should detail monitoring practices across firms as well as across workers and tasks within jobs and firms. This type of information is scarce since most data sets do not collect information on the intensity of monitoring within an establishment (plant or firm) and simply limit their inquiries to whether incentives and monitoring are used at all across the wide variety of jobs and tasks that take place within an establishment.

To the best of our knowledge, only a few descriptive studies have offered qualitative results from settings where managers coordinate incentives and monitoring. The most clear example that we are able to find in the existing literature is Hueth et al. (1999) who study contractual relations between growers and distributors in California fruit and vegetable markets. Hueth et al. (1999) show that market intermediaries coordinate incentives (mainly pay proportional to market price) with field visits and load measurement. ${ }^{8}$ Another example is Gittell (2000), a case study that compares incentives and monitoring practices in American Airlines and Southwest Airlines. The former airline differs from the latter in that they set stronger individual incentives along with stronger individual monitoring while Southwest combines softer (group) incentives with informal peer monitoring. Finally, well-known business case studies offer rich examples (such as the typist in General Electric) where strong incentives without strong monitoring are clearly far from optimal.

The main empirical implication of our model is that managers must provide higher incentives in those tasks that they choose to monitor more closely. This is because of the complementarity that emerges endogenously within the model between incentives and monitoring activities through the attention allocation constraint of the principal. To the best of our knowledge, there are no empirical papers exploiting variation from large data sets that provide direct evidence of this implication. Ideally we would test these predictions with a data set that details monitoring and incentives within a firm across all its workers and jobs for some extended period of time with an exogenous change in monitoring technology or regulation that impacts the cost of monitoring workers and tasks during the period of observation.

Despite the lack of information on monitoring, many papers document the relation between contractibility and incentive provision in agency relationships without taking into account differences in monitoring practices across firms and jobs. These papers therefore must implicitly assume a uniform level of monitoring across jobs and tasks that may bias their empirical estimates of the relation between contractibility and

\footnotetext{
8 Their paper provides their data in a table and it is easy to show a positive correlation between incentive provision and the number of field visits and own-measurement practices.
} 
incentives as well as the relation between incentives and productivity. This omitted variable econometric problem must be then biasing their estimates down towards zero.

Take now as example the implications from Ackerberg and Botticini (2002). They argue that in a regression containing incentive provision as dependent variable and job characteristics in the right hand side, coefficients are likely to be biased due to endogenous matching between employees and jobs, that is, a plausible correlation between the independent variables and the error term. Our paper adds to the empirical content of Ackerberg and Botticini (2002) in that we show that incentive provision and job design may be conditioned by the degree of monitoring intensity available to the principal and therefore part of this unobserved (to the econometrician) correlation between independent variables and the error term may be due to omitted variables such as monitoring technologies and monitoring effort that are endogenous and simultaneously determined with incentives and job characteristics. Having data on the principal's allocation of monitoring to different jobs and tasks may help with the endogeneity problem at hand.

It is important to note though that our implications predict estimates biased toward zero due to the presence of omitted variables while Ackerberg and Botticini (2002) predict a positive bias due to self-selection of employees into their most adequate jobs. Therefore it is difficult to know ex-ante in which direction the net bias will go.

On the other hand, our results show that under strong complementarities across tasks it will always be optimal to balance incentives and attention across tasks. However, when tasks are independent, the principal may optimally choose to offer an unbalanced incentive provision (and attention allocation) across tasks. An extreme result from our model is that under increasing returns to scale in the production or monitoring technology the principal may mute incentives in some relevant tasks (to their profit function) and focus their monitoring and incentives in a subset of tasks that define a job. Therefore, another way to test the implications of our model would be to observe monitoring activities across tasks before and after an employer decides to mute incentives in some tasks and increase them in others. A positive correlation between incentives and monitoring activities for a given task within a contract would be consistent with the testable implications in our model. We may find consistent evidence (not definite tests though) in papers that studied contracts muting incentives for some of the tasks defining jobs (see Chiappori and Salanie 2003 for a list of a few examples).

Similarly, our model predicts that monitoring and incentives in all tasks will move in the same direction as long as the total amount of attention increases. Otherwise, our model assumes that incentives and monitoring across tasks within a job should be negatively correlated. For example, the adoption of on-board computers (OBC hereafter) in trucking, as studied in Baker and Hubbard (2003), may have lowered the cost of monitoring across all tasks and therefore increased the total attention capacity. In that case, our model would predict an increase in incentives across all tasks. If the adoption of $\mathrm{OBC}$ had lowered monitoring costs in only a few tasks within a job (driving routes versus truck maintenance) our predictions would indicate that incentives in those tasks affected by $\mathrm{OBC}$ adoption may increase and incentives in other tasks may decrease. Finally, if different firms specialized in different services and therefore jobs differ in their task composition across firms, the introduction of OBC may 
increase incentives more sharply in those firms where jobs are composed by fewer tasks. Unfortunately, these predictions are not testable with the data set used in Baker and Hubbard (2003) since monitoring and incentive information at the job and task level are not available.

A last set of prediction comes from results in Sect. 4 which suggest that, in the case of increasing returns, one could see sharp changes in specialization patterns within companies for relatively small changes in prices of output for different tasks. To test this prediction, the empirical researcher would need a data set containing information on incentives and monitoring intensities across a set of firms within an industry and observe a change in the relative output price of different tasks. Our model specifically predicts that specialization of the firms in the data set would happen at the same time that the intensity of both incentives and monitoring increased for those tasks that the firm is choosing to specialize into. A classical example consistent with this prediction is the pin factory of Adams Smith and the relevance of market size for the extent of the division of labor. As market size increases, the gains of worker specialization in either task increases and therefore the relative output price increases (away from 1 in our model). According to Smith, this moves pin factories to reorganize their workers into more specialized jobs and therefore only reward them for their performance in a subset of the tasks that they carried before. Smith's accounting failed to provide us with information on the changes in monitoring that came along specialization in pin factories located in larger markets and therefore we cannot test whether our model is relevant in that context.

Our other empirical implications are also consistent with other stylized facts in the existing literature. Other papers have documented cases when the cost of monitoring has gone down due to an exogenous factor such as a change in technology. As mentioned above, Baker and Hubbard (2003) show that the introduction of OBC lowered the costs of monitoring (and presumably the amount of monitoring increased) in the trucking industry and increased the amount of outsourcing with high-powered incentives for certain types of hauls. If attention allocated to a task is correlated with the level of investment in monitoring technology in that particular task, their results are consistent with our testable implications. In another paper, Lerner and Malmendier (2008) study the relation between contractibility and the design of contracts in biotechnology research. In their setting, financing firms worry that research firms use their funding to pursue side projects. They find that when actions are not contractible an option contract becomes optimal since the threat of termination strengthens the incentives of the research firm. Finally, we find more supporting evidence in Slade (1996) who empirically examines contracts between private, integrated oil companies and their service stations in Vancouver. She shows that variation in characteristics of a task (possibly correlated with contractibility) optimally changes compensation scheme for other tasks.

All of these papers offer evidence that indirectly support our results in that a quasi-exogenous shock in the cost of monitoring (a change in the monitoring technology) affects the contracting scenario and changes the strength of incentives optimally offered. Despite this, there is no direct evidence relating information on monitoring practices (at firm, job or task level) with incentive provision, and therefore there is not yet a direct test of our model. 


\section{Concluding remarks}

In this paper, we introduce managerial attention allocation in optimal incentive contracts by extending the model in Holmstrom and Milgrom (1991). In our model, managers are constrained in the total amount of monitoring capacity that they can allocate across tasks. The allocation of attention across tasks becomes a managerial problem with clearly defined trade-offs. Specifically, more attention allocated to one task implies less monitoring in other tasks. When managers allocate more attention to a given task, the worker's effort on that task becomes more contractible and therefore the manager optimally provides stronger incentives on the realization of that same task.

We find that managers allocate the same level of attention and provide the same incentives for both tasks when production and monitoring technologies exhibit decreasing returns to scale. When relaxing these initial conditions and allowing for the presence of increasing returns for both tasks in either production or monitoring, the symmetry of the results disappears and we find that managers may optimally concentrate all their attention and provide incentives only for one of the tasks. After that, we introduce asymmetries in the way different tasks enter the production and monitoring technologies. As a result of this asymmetry, we find that managers optimally allocate different amounts of attention to different tasks and provide different incentives to different tasks.

These findings provide an alternative and complementary explanation for the use of simple unidimensional contracts in multi-tasking settings. In cases when managers can act upon and change their monitoring activities (through direct monitoring or investment in new monitoring technologies), they may coordinate their attention allocation decisions and incentive contracts. In particular, they may choose to concentrate all their attention and incentives in a few tasks and leave some others unmentioned in the contracts at use. This result from our model is consistent with the fact that most jobs are multidimensional and yet managers and principals use simple contracts that concentrate in only a few of the tasks that compose the job.

A straightforward extension of the model at hand would be to generalize the case of two tasks presented here into $n$ tasks. We believe though that our main results will not change qualitatively, and we foresee that the analysis and testable implications presented in this paper are easily generalizable and that they do not change when the number of tasks defining the job increases. Another possible extension is to adopt other forms of monitoring technologies that resemble more closely the monitoring tools that managers have available. Future lines of research are to include complementarities between tasks in monitoring and study the implications of managerial attention allocation for other incentive problems and vertical integration decisions.

Open Access This article is distributed under the terms of the Creative Commons Attribution License which permits any use, distribution and reproduction in any medium, provided the original author(s) and source are credited.

\section{References}

Ackerberg D, Botticini M (2002) Endogenous matching and the empirical determinants of contract form. J Polit Econ 110(3):564-591 
Bacchetta P, Van Wincoop E (2006) Infrequent portfolio decisions: a solution to the forward discount puzzle. Am Econ Rev (forthcoming)

Bajari P, McMillan R, Tadelis S (2007) Auctions versus negotiations in procurement: an empirical analysis. $\mathrm{J}$ Law Econ Org (forthcoming)

Baker GP (1992) Incentive contracts and performance measurement. J Polit Econ 100(3):598-614

Baker GP, Gibbons R, Murphy KJ (1994) Subjective performance measures in optimal incentive contracts. Q J Econ 109(4):1125-1156

Baker GP, Hubbard TN (2003) Make versus buy in trucking: asset ownership, job design, and information. Am Econ Rev 551-572

Chiappori P-A, Salanie B (2003) Testing contract theory: a survey of some recent work. In: Dewatripont M, Hansen L, Turnovsky P (eds) Advances in economics and econometrics-theory and applications, Eighth World Congress, Econometric Society Monographs, Cambridge University Press, Cambridge, pp 115-149

Corts K (2007) Teams vs. individual accountability: solving multi-task problems through job design. Rand J Econ 38(2):468-480

Demougin D, Fluet C (2001) Monitoring versus incentives. Eur Econ Rev 45:1741-1764

Englmaier F, Wambach A (2007) Optimal incentive contracts under inequity aversion. Mimeograph

Fehr E, Klein A, Schmidt KM (2007) Fairness and contract design. Econometrica 75:121-154

Fehr E, Schmidt KM (2003) Theories of fairness and reciprocity-evidence and economic applications. In: Dewatripont M, et al (eds) Advances in economics and econometrics, Eighth World Congress of the Econometric Society, vol 1, Cambridge University Press, Cambridge, pp 208-257

Gifford S (2004) To make or buy: an allocation of attention. Contrib Theor Econ 4(1) (article 2)

Gittell JH (2000) Paradox of coordination and control. Calif Manage Rev 42(3):101-116

Hart O, Moore J (2004) Agreeing now to agree later: contracts that rule out but do not rule. In: NBER Working Paper No. 10397

Hart O, Moore J (2008) Contracts as reference points. Q J Econ 123(1):1-48

Hennart J-F (1993) Explaining the swollen middle: why most transactions are a mix of 'market' and 'hierarchy'. Org Sci 4:529-547

Holmstrom B (1979) Moral hazard and observability. Bell J Econ 10(1):74-91

Holmstrom B, Milgrom P (1991) Multitask principal-agent analyses: incentive contracts, asset ownership, and job design. J Law Econ Org 7(0):24-52

Holmstrom B, Milgrom P (1994) The firm as an incentive system. Am Econ Rev 84(4):972-991

Hueth B, Ligon E, Wolf S, Wu S (1999) Incentive instruments in fruit and vegetable contracts: input control, monitoring, measuring, and price risk. Rev Agric Econ 21(2):374-389

Kessler A (2004) Optimal auditing in hierarchical relationships. J Institutional Theor Econ 160:210-231

Khalil F, Lawarree J (1995) Input versus output monitoring: who is the residual claimant? J Econ Theory 66:139-157

Lazear E (2006) Speeding, terrorism, and teaching to the test. Q J Econ 121(3):1029-1061

Lerner J, Malmendier U (2008) Contractibility and the design of research agreements. Am Econ Rev (forthcoming)

Lucas RE (1978) On the size distribution of business firms. Bell J Econ 10(2):508-523

Luo Y (2008) Consumption dynamics under information processing constraints. Rev Econ Dyn 11:366-385

MacDonald G, Marx LM (2001) Adverse specialization. J Polit Econ 109(4):864-899

Mackoviak B, Wiederholt M (2007) Optimal sticky prices under rational inattention. Am Econ Rev (forthcoming)

Mankiw G, Reis R (2002) Sticky information versus sticky prices: a proposal to replace the New Keynesian Phillips Curve. Q J Econ 117(4):1295-1328

Mankiw G, Reis R (2003) Sticky information: a model of monetary non-neutrality and structural slumps. In: Aghion P, Frydman R, Stiglitz J, Woodford M (eds) Knowledge, information, and expectations in modern macroeconomics: In Honor of Edmund S. Phelps. Princeton University Press, Princeton, pp 64-86

Mondria J (2006) Financial contagion and attention allocation. University of Toronto Working Paper

Reis R (2006) Inattentive consumers. J Monetary Econ 53(8):1761-1800

Reis R (2006) Inattentive producers. Rev Econ Stud 73(3):793-821

Rosen S (1982) Authority, control, and the distribution of earnings. Bell J Econ 13(2):311-323

Shavell S (1979) Risk sharing and incentives in the principal and agent relationship. Bell J Econ 10(1): $55-73$ 
Sims C (2003) Implications of rational inattention. J Monetary Econ 50(3):665-690

Sims C (2006) Rational inattention: beyond the linear-quadratic case. Am Econ Rev Papers Proc 96(2): $158-163$

Slade M (1996) Multitask agency and contract choice: an empirical exploration. Int Econ Rev 37(2):465486

Van Nieuwerburgh S, Veldkamp LL (2007a) Information acquisition and portfolio under-diversification. Working paper

Van Nieuwerburgh S, Veldkamp L (2007b) Information immobility and the home bias puzzle. J Finance (forthcoming)

Wernerfelt B (2007) Renegotiation facilitates contractual incompleteness. J Econ Manage Strategy 16(4):893-910 